Am J Cardiol. 2018 November 01; 122(9): 1578-1587. doi:10.1016/j.amjcard.2018.07.012.

\title{
Primary and Secondary Diastolic Dysfunction in Heart Failure With Preserved Ejection Fraction
}

\author{
Marco Giuseppe Del Buono, MD ${ }^{\mathrm{a}, \mathrm{c}}$, Leo Buckley, PharmD ${ }^{\mathrm{b}}$, and Antonio Abbate, MD, $\mathrm{PhD}^{\mathrm{a},{ }^{*}}$ \\ aVCU Pauley Heart Center, Wright Center for Clinical and Translational Research, Richmond, \\ Virginia \\ bDivision of Cardiovascular Medicine, Brigham and Women's Hospital, Harvard Medical School, \\ Boston, Massachusetts \\ 'Department of Cardiovascular Medicine, Catholic University, Rome, Italy.
}

\section{Abstract}

\begin{abstract}
Approximately $50 \%$ of patients with symptoms and signs of heart failure have a left ventricular ejection fraction (LVEF) $\geq 50 \%$ and are often simply referred to as "heart failure with preserved EF', 'HFpEF'. Many of such patients have HF secondary to specific cardiac conditions (i.e., valvular or pericardial disease) in which the symptoms and signs occur despite the LVEF being preserved due to diastolic dysfunction secondary to the underlying disease (secondary $H F p E F$ ), differently from those HFpEF patients in which the impaired LV filling is due to a primary diastolic dysfunction (primary HFpEF). When primary HFpEF patients are properly diagnosed, they appear to have a milder form of HF with a lower cardiovascular mortality compared with $\mathrm{HFrEF}$ and secondary HFpEF population, but a risk of HF hospitalization that is significantly higher than patients with similar cardiovascular risk factors but without the diagnosis of HFpEF. We herein review the diagnostic approach to HFpEF and present a differential diagnosis of HFpEF in a primary and secondary form.
\end{abstract}

\begin{abstract}
Heart failure (HF) is a syndrome characterized by specific symptoms (i.e., shortness of breath, exercise intolerance, and swelling) and signs (i.e., pulmonary congestion, lower extremity edema, and elevated jugular venous pressure) caused by functional and/or structural impairment of cardiac function(s) that compromise the capacity of achieving a proper diastolic filling and/or keeping an adequate cardiac output, either at rest or during exertion. ${ }^{1}$ Moreover, patients with HF often have alterations in the respiratory, vascular, and muscular systems which contribute to the symptomatology and disability of the condition, but what defines HF, and differentiates this from other noncardiac chronic conditions with similar debilitating symptoms, is the impairment in cardiac function.
\end{abstract}

\footnotetext{
"Corresponding author: Tel: +1 (804) 828-0513; fax: +1 (804) 82-86765., antonio.abbate@ vcuhealth.org (A. Abbate). Disclosures

The authors have no conflicts of interest to disclose.

Supplementary materials

Supplementary data associated with this article can befound, in the online version, https://doi.org/10.1016/j.amjcard.2018.07.012.
} 


\section{Why is Determining the Ejection Fraction (EF) So Important in HF?}

HF is historically classified according to the left ventricle EF (LVEF), allowing identification of a more homogenous group of patients. ${ }^{2}$ Nearly half of patients with HF have systolic dysfunction defined as LVEF $<40 \%$, HF with reduced EF (HFrEF) or systolic HF. ${ }^{1,3}$ In randomized clinical trials in HFrEF, angiotensin-converting enzyme inhibitors, angiotensin receptor blockers, beta-adrenergic receptor blockers, aldosterone antagonists and, in selected populations, isosorbide dinitrate and/or hydralazine, angiotensin receptor blocker and/or neprilysin inhibitor and ivabradine reduce all-cause mortality and/or hospitalizations for HF. ${ }^{1,3}$

However, more challenging is the definition and estimation of prevalence and prognosis of those patients in whom the HF syndrome occur despite preserved EF (defined as LVEF $250 \%$ ). According to case series, these patients represent approximately $50 \%$ (range $22 \%$ to 73\%) (Figure 1) of those with HF and this wide variation is primarily attributed to the clinical setting, characteristics of the studied population, the different cut-off values of normal EF, the definition applied to define HF, how detailed the protocol was to exclude other causes of HF (i.e., valvular heart disease), and the potential for misdiagnosis (i.e., noncardiac disease). The prognosis of patients with HF and LVEF $\geq 50 \%$ also varies largely across studies, likely due to the same reasons.

Given the important role of LVEF $<40 \%$ in identifying a relatively homogenous cohort of subjects (HFrEF) and considering the large heterogeneity in prevalence, prognosis and response to treatment in patients with $\mathrm{HF}$ and LVEF $>40 \%$, it is important to determine the reasons for this heterogeneity and phenotypically characterize patients with HF and LVEF $>40 \%$, and in particular those with LVEF $\geq 50 \%$. These patients are indeed often referred to as having HF with preserved EF (HFpEF), in contrast to those with HFrEF. Labeling every patient with HF symptoms and LVEF $\geq 50 \%$ as having HFpEF, however, suggest that a common pathophysiologic denominator exists across these patients, whereas in reality, as described, the only defining factor is the negative connotation of lacking significant systolic dysfunction. Labeling all patients with HF symptoms and LVEF $250 \%$ as HFpEF may however be insufficient to guide the diagnostic and therapeutic approach. Cases of HFpEF secondary to identifiable myocardial, valvular or pericardial diseases (secondary $H F P E F$ ) may indeed benefit from a tailored diagnostic and therapeutic approaches and prognostication, whereas the remaining cases (primary $H F p E F$ ) are usually due to common metabolic and hemodynamic risk factors, milder in its form, and a need for additional research in terms of therapeutic strategies (Figure 1).

Patients with HF and EF between $40 \%$ and $49 \%$ have been classified as HFpEF in the past for clinical trials purpose, however, while there is limited prospective clinical data, the prognosis of these patients appears intermediate between those with reduced $(<40 \%)$ and preserved ( $250 \%) \mathrm{EF},{ }^{1,3}$ and in absence of dedicated clinical trials most cardiologists recommend treating these patients as HFrEF. Patients with HF and reduced EF who recover the $\mathrm{EF}$ above $50 \%$ (after reversible cardiac injury or following medical therapy) are considered as a distinct HF phenotype than those with HFpEF (HF with recovered EF) 
because those patients that experience a recovery of EF while on HF medications have a more favorable clinical prognosis and are generally continued on such medications. ${ }^{5}$

\section{The current definition of HFpEF}

The most recent definition by the European Society of Cardiology (ESC) has clarified that besides symptoms and signs of HF and a LVEF $250 \%$, documentation of elevation of cardiac biomarkers (brain natriuretic peptide [BNP] $>35 \mathrm{pg} / \mathrm{ml}$ or N-terminal pro BNP $>125 \mathrm{pg} / \mathrm{ml}$ ) and at least one feature of structural heart disease and/or diastolic dysfunction is needed. ${ }^{3}$ Structural and functional alterations suggestive of elevated LV filling pressure are atrial adverse remodeling (left atrial volume index $>34 / \mathrm{ml}^{2}$ ) and E/E'>14 (at rest), that appear to correlate with invasive measurements of pulmonary capillary wedge pressure $\geq 15$ or left ventricular end diastolic pressure $>16 \mathrm{~mm} \mathrm{Hg}$. Alterations correlating with impaired relaxation and reduced compliance include mean E' $<9 \mathrm{~cm} / \mathrm{s}$ (septal E' $<7 \mathrm{~cm} / \mathrm{sec}$, lateral E' $<10 \mathrm{~cm} / \mathrm{sec}$ ), E/E'>14 (with exercise) and increased LV mass index 295 (females) or $\geq 115$ (males) $\mathrm{g} / \mathrm{m}^{2}$ ). ${ }^{1,3,4}$ Other supportive findings are pulmonary arterial hypertension (peak tricuspid regurgitation velocity $>2.8 \mathrm{~m} / \mathrm{sec}$ ) and an abnormal LV longitudinal systolic strain function. Elevated of BNP or NT-proBNP levels are mandatory for HFpEF diagnosis according to ESC 2016 definition, however, the sensitivity outside of the acute setting (i.e., chronic stable HF) has been questioned, especially in obese and non-Caucasian patients. ${ }^{6,7}$

At difference with previous HFpEF definitions, including the American College of Cardiology Foundation/American Heart Association 2013 definition, ${ }^{1}$ the ESC 2016 definition allows to differentiate in those patients with HF and LVEF $\geq 50 \%$, between those with evidence of functional and/or structural cardiac abnormalities, from the other patients that did't show any of these abnormalities and may be suffering from noncardiac diseases. Neither the American College of Cardiology Foundation/American Heart Association 2013 definition, ${ }^{1}$ the ESC 2016 definition ${ }^{3}$ attempt a differentiation between primary versus secondary forms of HFpEF.

\section{Are "HFpEF" and "diastolic HF" One and the Same?}

Although the ESC definition has added specificity to the HFpEF diagnosis and helps distinguishing HFpEF from noncardiac illnesses, the importance of excluding specific underlying cardiac causes may have not been sufficiently addressed, and this may perhaps be leading to possible overestimation of the prevalence of primary HFpEF in routine clinical practice. There are indeed cardiac conditions (i.e., pericardial effusion or constriction, restrictive cardiomyopathies, and severe valvular diseases) that can cause symptoms of $\mathrm{HF}$ in presence of a normal LVEF but do not constitute an intrinsic abnormality in the myocardial diastolic function but rather a dysfunction secondary to an underlying problem that requires an independent assessment. Therefore, while approaching HFpEF, one should address whether symptoms can be attributed to a dysfunction in cardiac filling (diastolic dysfunction) secondary to a specific identifiable cause, secondary $H F p E F$ (i.e., severe valvular disease, constrictive pericarditis, restrictive or hypertrophic cardiomyopathy, tachyarrhythmia, high-output HF, right HF). Many of these cardiac conditions would indeed benefit from a specific diagnosis because requiring a targeted therapy (Figure 2, Table 1). ${ }^{8-10}$ 


\section{HFpEF Due to Primary Diastolic Dysfunction or Primary HFpEF}

The diagnosis of primary HFpEF, as clinical entity, should be reserved for those cases in which there is a primary diastolic dysfunction leading to HF, as result of interaction of multiple risk factors such as hypertension, aging, systemic inflammatory status or metabolic disorders, but without any specific underlying cause and in which there is currently no specific medical or surgical therapies (Figure 3). Patients with primary $H F p E F$ represent a specific "phenotype" and are usually middle-age or older subjects, more often women, often with a history of hypertension, obesity, diabetes mellitus (DM) type 2, chronic kidney disease, and atrial fibrillation. ${ }^{11-13}$ Obstructive coronary artery disease may co-exist, but is less common than in the HFrEF population. When HFrEF and secondary causes of HFpEF have properly been excluded, a simple score, the $H_{2} F P E F$ score, has been proposed to estimate the probability of underlying HFpEF in patients with unexplained exertional dyspnea. This score utilized 6 clinical and echocardiographic variables (obesity [BMI>30 $\left.\mathrm{kg} / \mathrm{m}^{2}\right]-3$ points, atrial fibrillation- 2 points, age $>60$ years- 1 point, treatment with 2 or more antihypertensive drugs-1 point, E/e' $>9-1$ point, pulmonary artery systolic pressure $>35 \mathrm{~mm}$ Hg-1 point) leading to a weighed sum ranging from 0 to 9 . A high score ${ }^{6-9}$ is associated with a higher likelihood of HFpEF diagnosis, while a low score (0 to 1) suggests noncardiac causes for the symptoms, and an intermediate score ${ }^{2-5}$ identifies patients in which further evaluations are needed to reach a definitive diagnosis. ${ }^{14}$ Of note, with the exception of the Doppler criteria, which is also only lightly weighed, the remaining criteria seem to lack specificity for HFpEF, nevertheless they remain highly predictive of HFpEF in population studies due to epidemiologic associations, and thus, this simple score may prove valuable as a way to take into account the risk factors for $\mathrm{HFpEF}$ and lead to a pretest probability of HFpEF.

\section{HFpEF Secondary to an Identifiable Cause or Secondary HFpEF}

Patients presenting with HF and LVEF $\geq 50 \%$ and symptoms secondary to an identifiable cause of diastolic dysfunction are considered secondary HFpEF, also referred to as ' $\mathrm{HFpEF}$ mimics'. Such conditions have unique clinical presentations and trajectories, and may respond to specific treatments dependent on the underlying causes (Figure 2, Table 1, Supplemental Table 1). ${ }^{8,9}$ Among secondary forms of HFpEF is possible to identify secondary forms with identifiable and treatable causes (i.e., valvular heart disease, pericardial disease) and secondary identifiable but untreat-able causes (i.e., restrictive cardiomyopathy following radiotherapy). These conditions need to be promptly identified through a comprehensive history, physical examination, electrocardiogram, echocardiography and often cardiac magnetic resonance, and treated according to their specific pathophysiology.

Therefore, while the primary $H F p E F$ and the secondary $H F p E F$ share the presence of diastolic dysfunction and preservation in LVEF, and may experience an improvement in symptoms with diuretics, prognosis can differ substantially, and targeted therapies may exist in some secondary cases. 


\section{What Causes Primary HFpEF?}

The central pathophysiological disturbance in primary $H F p E F$ is diastolic dysfunction. The term diastolic dysfunction refers to changes in ventricular diastolic properties that have an adverse effect on ventricular filling and stroke volume. ${ }^{9,15-17}$ The main determinants of diastolic dysfunction are impaired LV relaxation (impaired lusitropy) and/or increase LV stiffness (reduced compliance). ${ }^{9}$ These conditions often co-exist and result in increase in end-diastolic pressure, impaired ventricular filling, and therefore decreased end-diastolic volume, and stroke volume, as shown by changes of the ventricular pressure volume loop. LVEF is normal and occasionally higher than normal, yet there can be a reduction in the stroke volume secondary to a shift of left ventricle end-diastolic volume to the left of pressure-volume loop, and the cardiac output may be decreased.

Hypertension is the most common condition associated with primary $H F p E F$, and is historically considered the main driver of diastolic dysfunction, ${ }^{16}$ although it is important to note that the diagnosis of primary $H F p E F$ should be made when symptoms persist after treatment of elevated blood pressure levels. HFpEF in the patient with hypertension should therefore be differentiated from the symptoms of uncontrolled hypertension.

Traditionally, it is proposed that primary $H F p E F$ is the result of chronic pressure overload leads to adaptively concentric left ventricular hypertrophy (LVH) and fibrosis resulting in impaired compliance and diastolic dysfunction. ${ }^{18} \mathrm{LVH}$ is present in some patients with primary $H F p E F$, however, a significant proportion of patients with primary $H F p E F$, does not have $\mathrm{LVH}$ defined as a LV mass $>115 \mathrm{~g} / \mathrm{m}^{2}$ in men and $>95 \mathrm{~g} / \mathrm{m}^{2}$ in women, but rather have concentric remodeling. ${ }^{18-19}$ The clinical prognostic values of concentric remodeling and concentric LVH appear to be similar. Extracellular fibrosis is also increased in primary $H F p E F$ patients as it compares with asymptomatic hypertensive controls with LVH. ${ }^{19}$ In addition to hypertension, is emerging that other co-morbidities, often present in patients with primary $H F p E F$ (e.g., obesity, DM, metabolic syndrome, lung disease, smoking, systemic oxidative stress, and iron deficiency), promote microvascular endothelial inflammation, myocardial inflammation, fibrosis, increases in oxidative stress and alterations in cardiomyocyte signaling pathways, resulting in global cardiomyocyte stiffness, cardiomyocyte hypertrophy, and impaired active relaxation. ${ }^{8}$ Microvascular dysfunction may also involve coronary vessels resulting in reduced microvascular density and coronary flow reserve in primary $H F p E F^{8,20}$ and may explain the limitations in LV systolic and diastolic reserve during exercise.

Furthermore, although HF requires a central impairment to be present, peripheral abnormality in the skeletal muscle energetics have been consistently demonstrated in patients with HF, both HFrEF and HFpEF, and appear to be an important determinant of fatigability and exercise intolerance ${ }^{21}$ Figure 3 provides a schematic representation of the mechanisms leading to diastolic dysfunction in HFpEF. 


\section{Why is Important to Recognize HFpEF?}

Despite the clear prognostic value of LVEF in HF, the presence and severity of symptoms and signs of HF identifies patients at increased risk of adverse cardiovascular (CV) outcomes, independent of LVEF values. Therefore, it is important to recognize that HF presents across the spectrum of LVEF values. ${ }^{22}$ When population studies categorize patients with HF in 2 exclusive categories based on LVEF, the prognosis of HFpEF appears to be not better than HFrEF. ${ }^{12,12,23}$ It is worth noting, however, that this classification may be fraught by diagnostic uncertainties regarding the overall prevalence of primary versus secondary forms of $H F p E F$, and it is very likely that secondary $H F p E F$ such as valvular heart disease, hypertrophic, and infiltrative cardiomyopathies may be the drivers of worse prognosis in the overall HFpEF cohort. $^{8}$ )

When attempting to define prognosis, it is therefore important to distinguish between primary and secondary HFpEF. Indeed, in retrospective, observational, and population studies, in which HFpEF is diagnosed based on LVEF values in the chart and without any attempt to exclude secondary forms, the rates of hospitalization and death in patients who have HFpEF is reported to be similar to those in patients who have presumed HFrEF. ${ }^{12,13}$ In clinical trials targeting primary HFpEF and prospective studies, in which the diagnosis was in general made with an attempt to exclude secondary causes, the $\mathrm{CV}$ outcomes were significantly better in patients with HFpEF than those with HFrEF. ${ }^{24,25}$ This is in line with the known prognostic value of LVEF, and therefore it can be concluded that patients with HFpEF (once secondary HFpEF forms have been excluded) suffer from a milder form of HF associated with a lower CV mortality, as compared with HFrEF, and not much different from that of patients with hypertension and multiple risk factors. Vice versa, the risk of HF hospitalization differs substantially between hypertensive patients without pre-existing $\mathrm{HF}$ and those with (primary) $\mathrm{HFpEF}$ with the latter being at a much higher risk of $\mathrm{HF}$ hospitalization (Figure 4). ${ }^{26-28}$

\section{The Secondary Causes of HFpEF That You Can't Afford to Miss}

\section{Valvular heart disease}

The increased afterload in patients with severe aortic stenosis leads to a compensatory adaptive concentric hypertrophy causing diastolic dysfunction and HF, independent of EF. Cardiac remodeling in response to volume overload in chronic mitral and aortic regurgitation is of eccentric hypertrophy, a pattern that is rarely seen in primary HFpEF. Mitral stenosis creates a transmitral gradient at rest or with exertion, left atrial hypertension and, over time, right-sided HF.

Failure to identify valvular heart disease as the cause of HF syndrome can lead to a delay in surgical or transcatheter replacement and/or repair and avoidable morbidity and mortality. ${ }^{29}$

\section{Hypertrophic cardiomyopathy}

Hypertrophic cardiomyopathy may present different forms of LVH and especially in the concentric form, it may be difficult to differentiate from hypertensive heart disease. Because is associated with an increased risk of sudden cardiac death, implantable cardioverter- 
defibrillator implantation is needed in high-risk patients. Cardiac magnetic resonance imaging is very helpful as it can highlight the hypertrophy phenotype, evaluate for outflow tract obstruction and mitral regurgitation, and for myocardial scarring. ${ }^{30}$

\section{Constrictive pericarditis}

Constrictive pericarditis is the result of a reduced pericardial compliance often associated with a thickened or calcified pericardium. In patients with symptoms and signs of HF and history of pericarditis, trauma, radiation therapy, cardiac surgery, or a systemic disease that affects the pericardium (i.e., tuberculosis, connective tissue disease, and malignancy), constrictive pericarditis should be always considered. Echocardiography can detect an exaggerated respiratory interdependence of the ventricles and high $\mathrm{E}$ velocity of right and left ventricle inflow despite a high tissue Doppler velocity at mitral annulus (E'). Medial mitral annular E' velocity is usually greater than the lateral mitral annular E' in constrictive pericarditis, in contrast with other causes of $\mathrm{HF}^{31}$ Diuretics, anti-inflammatory therapies, and pericardiectomy may be required.

\section{Restrictive cardiomyopathy}

Restrictive cardiomyopathy is characterized by severely impaired myocardial compliance and diastolic dysfunction and normal $\mathrm{EF}^{32,33} \mathrm{LV}$ wall thickness is usually normal in patients with endomyocardial fibrosis, radiation-heart disease, iron overload cardiomyopathy and idiopathic restrictive cardiomyopathy, while is usually increased in patients with infiltrative forms (i.e., amyloidosis, and glycogen storage diseases). The diagnosis can be very challenging. The presence of a systemic disease that may involve the heart muscle (i.e., amyloidosis, sarcoidosis, and scleroderma) or specific risk factors (i.e., chest radiation therapy) significantly increase the likelihood of the disease. Cardiac magnetic resonance imaging is a very valuable diagnostic modality, but definitive diagnosis may require an endomyocardial biopsy. In absence of a curative approach for the underlying disease, the prognosis for restrictive cardiomyopathy is often grim. Symptomatic therapy generally includes diuretics for relief of congestion. Patients with severe restrictive cardiomyopathy often tolerate poorly beta-adrenergic receptor blockers, and do not respond to inotropes. ${ }^{32,33}$

\section{Regional wall motion abnormalities and/or LV dilatation with preserved EF}

Patients with large regional wall motion abnormalities and/or LV dilatation with globally preserved EF (i.e., after acute myocardial infarction or doxorubicin toxicity) should be treated as incipient-dilated cardiomyopathy, since after an initial insult cardiac remodeling can be a compensatory mechanism but also a precursor to systolic dysfunction and HFrEF. ${ }^{34}$

\section{Acute tachyarrhythmias}

In a setting of acute tachyarrhythmia, especially if underlying LV hypertrophy is present, diastolic function is impaired because of reduced ventricular filling time (diastole) and increased myocardial oxygen consumption leading to incomplete ventricular relaxation, and elevated filling pressures. These patients may, therefore, experience transient HF symptoms while having a normal EF with a significant clinical improvement after the restoring of sinus rhythm or heart rate control. Therefore, a clinical and echo-cardiographic reassessment 
should be repeated after the arrhythmia control, since also patients with primary HFpEF may experience HF exacerbation during an acute tachyarrhythmia. Differently, patients with long-standing tachyarrhythmias may develop a dilated cardiomyopathy with reduced EF. ${ }^{35}$

\section{High-output HF}

High-output HF is caused by a hyperdynamic state related to a reduction in systemic vascular resistances. The elevation of cardiac filling pressures is secondary to impairment in diastolic reserve related to the tachycardia and increased cardiac output. Often, there is also an expansion of plasma volume and neurohormonal activation that aggravate the $\mathrm{HF}$ syndrome. The most common causes of high-output HF are cirrhosis, severe anemia, thyrotoxicosis, and arteriovenous shunts. Morbid obesity has been also identified (when cardiac index is high $\left.>3.51 / \mathrm{min} / \mathrm{m}^{2}\right) .{ }^{36}$ High-output HF differs from primary $H F p E F$ because in HFpEF cardiac index values are usually normal or reduced at rest. Resolution of the causes leading to the low-systemic vascular resistance and high-output state can reverse the HF syndrome.

\section{HFpEF and Obesity}

Obesity is a common condition in HF especially in primary HFpEF, and deserves special consideration. Obese patients are more likely to have right ventricular failure and an uncontrolled hypertension resulting in higher pulmonary artery wedge pressure. ${ }^{37}$ It is currently unresolved whether obese HFpEF patients have a secondary form of HFpEF or, in alternative, are 2 conditions that co-exist. Caloric restriction-induced weight loss is effective in improving exercise capacity in obese HFpEF population that however occurs without any significant improvement in systolic and diastolic cardiac function, proposing obesity as a comorbid condition that further limits exercise capacity and that should be targeted to improve adiposity-induced exercise intolerance. ${ }^{38-40}$

\section{HFpEF and DM}

DM greatly increases the risk of $\mathrm{HF}^{41}$ Diabetic cardiomyopathy refers to structural and/or functional myocardial abnormalities in diabetic patients that are not attributable to other external factors such as coronary artery disease. In type $2 \mathrm{DM}$, insulin resistance, hyperinsulinemia, hyperglycemia and/or lipotoxicity, lead to pathophysiological myocardial adaptations that promote cardiac stiffness, hypertrophy, and fibrosis that are considered the substrate for the diastolic dysfunction leading to a primary form of HFpEF. Differently, patients with long-standing autoimmune type $1 \mathrm{DM}$ often show a different phenotype characterized by dilated cardiomyopathy and systolic dysfunction (HFrEF) ${ }^{41}$

\section{Unanswered Questions in HFpEF}

Considering the lack of gold standard diagnostic criteria for HFpEF, the exact incidence and prevalence of HFpEF in its primary and secondary forms are unknown. Once the secondary causes of HFpEF and other noncardiac conditions have been properly excluded from primary $H F p E F$, the exact prevalence of primary $\mathrm{HFpEF}$ is unknown and, given the difficulty in recruiting patients in clinical trials, is likely that prevalence of primary forms is 
lower than expected. ${ }^{42}$ Current algorithm suggests the use of multiple criteria to diagnose HFpEF, yet it is not known whether all the proposed criteria carry the same prognostic value and whether one or more criteria can identify responsiveness to a specific therapy. Moreover, given the frequent co-existence of co-morbid conditions in patients with HFpEF and the relatively high incidence of noncardiac death in patients with suspected HFpEF, it remains to be determined which intervention, if any, will reduce mortality in this cohort of patients. The lack of benefit of pharmacologic interventions on the 'hard' clinical outcomes in HFpEF has been attributed to the heterogeneity of the syndrome and perhaps inappropriate patient selection. It should be, however also considered that the cohorts of patients with primary HFpEF, when secondary HFpEF are excluded, have a relatively low-cardiac mortality, that is substantially lower than patients with HFrEF, and not much higher than hypertensive patients with similar risk factors. ${ }^{26-28}$ As such it may not be reasonable to expect for a treatment to reduce cardiac mortality in a clinical trial of $\mathrm{HFpEF}$ in duration of 2 to 3 years, but rather focus on HF specific end points such as reduction in readmissions and/or improvement in exercise capacity and quality of life. ${ }^{43}$

\section{Summary}

HFpEF is a clinical syndrome of shortness of breath and/or exercise intolerance secondary to the elevated LV filling pressures at rest or with exertion either due to intrinsic abnormalities in LV diastolic function - primary HFpEF - or due to cardiac conditions such as severe valvular heart disease, cardiomyopathy, pericardial disease, or arrhythmias-secondary HFpEF. Female sex, advanced age, hypertension, obesity, and DM, are risk factors for diastolic dysfunction and primary HFpEF. When assessing a patient with suspected HFpEF, it is important to distinguish between primary and secondary HFpEF since secondary causes may have unique clinical trajectories and specific treatments. It is also important to assess and treat co-morbidities that accompany HFpEF, in particular chronic obstructive lung disease and morbid obesity that often contribute to the symptom burden and disability. No specific treatment has been shown to reduce $\mathrm{CV}$ mortality in patients with primary $\mathrm{HFpEF}$ but treatments aimed at the relief of symptoms of congestion and of risk factors, such as hypertension and co-existing co-morbid conditions, improving HF-related clinical outcomes. Exercise training and caloric restriction are recommended, especially in obese $\mathrm{HFpEF}$ patients. By understanding the pathophysiologic mechanisms of HFpEF, one can hope to develop new therapeutic strategies for this condition.

\section{Supplementary Material}

Refer to Web version on PubMed Central for supplementary material.

\section{Acknowledgment::}

We would like to thank Akshay S. Desai, MD, MPH (Brigham and Women's Hospital, Harvard Medical School, Boston, MA, USA), Justin Canada, MS, CEP, Salvatore Carbone, MS, and Benjamin Van Tassell, PharmD (Virginia Commonwealth University, Richmond, VA, USA), Ross Arena, PhD, PT (University of Illinois, Chicago, IL, USA), and Francisco Jose Romeo, MD (Hospital Italiano, Buenos Aires, Argentina) for their contribution to the discussions that have inspired this review over the past several months, and Cristina Conte (Catholic University, Rome, Italy) for her help with the illustrations. 


\section{References}

1. Yancy CW, Jessup M, Bozkurt B, Butler J, Casey DE Jr, Drazner MH, Fonarow GC, Geraci SA, Horwich T, Januzzi JL, Johnson MR, Kasper EK, Levy WC, Masoudi FA, McBride PE, McMurray JJ, Mitchell JE, Peterson PN, Riegel B, Sam F, Stevenson LW, Tang WH, Tsai EJ, Wilkoff BL. American College of Cardiology Foundation; American Heart Association Task Force on Practice Guidelines. 2013 ACCF/AHA guideline for the management of HF: a report of the American College of Cardiology Foundation/American Heart Association Task Force on Practice Guidelines. Circulation 2013;128:e240-327. [PubMed: 23741058]

2. Borlaug BA, Redfield MM. Diastolic and systolic heart failure are distinct phenotypes within the heart failure spectrum. Circulation 2011;123:2006-2014. [PubMed: 21555723]

3. Ponikowski P, Voors AA, Anker SD, Bueno H, Cleland JG, Coats AJ, Falk V, González-Juanatey JR, Harjola VP, Jankowska EA, Jessup M, Linde C, Nihoyannopoulos P, Parissis JT, Pieske B, Riley JP, Rosano GM, Ruilope LM, Ruschitzka F, Rutten FH, van der Meer P. Authors/Task Force Members; Document Reviewers. 2016 ESC Guidelines for the diagnosis and treatment of acute and chronic heart failure: the task force for the diagnosis and treatment of acute and chronic heart failure of the European Society of Cardiology (ESC). Developed with the special contribution of the Heart Failure Association (HFA) of the ESC. Eur J Heart Fail 2016;18:891-975. [PubMed: 27207191]

4. Nagueh SF, Smiseth OA, Appleton CP, ByrdBF Dokainish H 3rd, Edvardsen T, Flachskampf FA, Gillebert TC, Klein AL, Lancellotti P, Marino P, Oh JK, Popescu BA, Waggoner AD.

Recommendations for the evaluation of left ventricular diastolic function by echocardiography: an update from the American Society of Echocardiography and the European Association of Cardiovascular Imaging. J Am Soc Echocardiogr 2016;29:277-314. 10.1016/j.echo.2016.01.011. [PubMed: 27037982]

5. Kalogeropoulos AP, Fonarow GC, Georgiopoulou V, Burkman G, Siwamogsatham S, Patel A, Li S, Papadimitriou L, Butler J. Characteristics and outcomes of adult outpatients with heart failure and improved or recovered ejection fraction. JAMA Cardiol 2016;1:510-518. [PubMed: 27434402]

6. Buckley LF, Canada JM, Del Buono MG, Carbone S, Trankle CR, Billingsley H, Kadariya D, Arena R, Van Tassell BW, Abbate A. Low NT-proBNP levels in overweight and obese patients do not rule out a diagnosis of heart failure with preserved ejection fraction. ESC Heart Fail 2018;5:372-378. 10.1002/ehf2.12235. Epub 2018 Jan 18.

7. Bajaj NS, Gutiérrez OM, Arora G, Judd SE, Patel N, Bennett A, Prabhu SD, Howard G, Howard VJ, Cushman M, Arora P. Racial differences in plasma levels of n-terminal pro-b-type natriuretic peptide and outcomes: the Reasons for Geographic and Racial Differences in Stroke (REGARDS) Study. JAMA Cardiol 2018;3:11-17. 10.1001/jamacardio.2017.4207. [PubMed: 29167879]

8. Redfield MM. Heart failure with preserved ejection fraction. N Engl J Med 2016;375:1868-1877. [PubMed: 27959663]

9. Abbate A, Arena R, Abouzaki N, Tassell BWVan, Canada J, Shah K, Biondi-Zoccai G, Voelkel NF. Heart failure with preserved ejection fraction: Refocusing on diastole. Int J Cardiol 2015;179:430 440. [PubMed: 25465302]

10. Desai AS, Jhund PS. After TOPCAT: what to do now in heart failure with preserved ejection fraction. Eur Heart J 2016;37:3135-3140. [PubMed: 27075872]

11. Owan TE, Hodge DO, Herges RM, Jacobsen SJ, Roger VL, Redfield MM. Trends in prevalence and outcome of heart failure with preserved ejection fraction. N Engl J Med 2006;355:251-259. [PubMed: 16855265]

12. Bhatia RS, Tu JV, Lee DS, Austin PC, Fang J, Haouzi A, Gong Y, Liu PP. Outcome of heart failure with preserved ejection fraction in a population-based study. N Engl J Med 2006;355:260-269. [PubMed: 16855266]

13. Dunlay SM, Roger VL, Redfield MM. Epidemiology of heart failure with preserved ejection fraction. Nat Rev Cardiol 2017.

14. Reddy YNV, Carter RE, Obokata M, Redfield MM, Borlaug BA. A simple, evidence-based approach to help guide diagnosis of heart failure with preserved ejection fraction. Circulation 2018 10.1161/CIRCULATIONAHA.118.034646 pii:CIRCULATIONAHA.118.034646 10.1161/ CIRCULATIONAHA.118.034646. 
15. Borlaug BA. Mechanisms of exercise intolerance in heart failure with preserved ejection fraction. Circ J 2014;78:20-32. [PubMed: 24305634]

16. Borlaug BA, Lam CS, Roger VL, Rodeheffer RJ, Redfield MM. Contractility and ventricular systolic stiffening in hypertensive heart disease: insights into the pathogenesis of heart failure with preserved ejection fraction. J Am Coll Cardiol 2009;54:410-418. [PubMed: 19628115]

17. Trankle C, Canada JM, Buckley L, Carbone S, Dixon D, Arena R, Van Tassell B, Abbate A. Impaired myocardial relaxation with exercise determines peak aerobic exercise capacity in heart failure with preserved ejection fraction. ESC Heart Fail 2017;4: 351-355. [PubMed: 28772034]

18. Shah AM. Ventricular remodeling in heart failure with preserved ejection fraction. Curr Heart Fail Rep 2013;10:341-349. [PubMed: 24097113]

19. Mordi IR, Singh S, Rudd A, Srinivasan J, Frenneaux M, Tzemos N, Dawson DK. Comprehensive Echocardiographic and Cardiac Magnetic Resonance Evaluation Differentiates Among Heart Failure With Preserved Ejection Fraction Patients, Hypertensive Patients, and Healthy Control Subjects. JACC Cardiovasc Imaging 2017. pii: S1936-878X(17)30617-4.

20. Obokata M, Reddy YNV, Melenovsky V, Kane GC, Olson TP, Jarolim P, Borlaug BA. Myocardial injury and cardiac reserve in patients with heart failure and preserved ejection fraction. $\mathrm{J}$ Am Coll Cardiol 20187 3;72:29-40. 10.1016/j.jacc.2018.04.039. [PubMed: 29957229]

21. Kitzman DW, Haykowsky MJ, Tomczak CR. Making the case for skeletal muscle myopathy and its contribution to exercise intolerance in heart failure with preserved ejection fraction. Circ Heart Fail 2017; 10 pii: e004281. [PubMed: 28705911]

22. Ahmed A, Aronow WS, Fleg JL. Higher New York Heart Association classes and increased mortality and hospitalization in patients with heart failure and preserved left ventricular function. Am Heart J 2006;151:444-450. [PubMed: 16442912]

23. Smith GL, Masoudi FA, Vaccarino V, Radford MJ, Krumholz HM. Outcomes in heart failure patients with preserved ejection fraction: mortality, readmission, and functional decline. J Am Coll Cardiol 2003;41:1510-1518. [PubMed: 12742291]

24. Meta-analysis Global Group in Chronic Heart Failure (MAGGIC). The survival of patients with heart failure with preserved or reduced left ventricular ejection fraction : an individual patient data meta-analysis. Eur Heart J 2012;33:1750-1757. [PubMed: 21821849]

25. Lam CSP, Gamble GD, Ling LH, Sim D, Leong KTG, Yeo PSD, Ong HY, Jaufeerally F, Ng TP, Cameron VA, Poppe K, Lund M, Devlin G, Troughton R, Richards AM1, Doughty RN. Mortality associated with heart failure with preserved vs. reduced ejection fraction in a prospective international multi-ethnic cohort study. Eur Heart J 2018;39:1770-1780. 10.1093/eurheartj/ ehy005. [PubMed: 29390051]

26. ALLHAT Officers and Coordinators for the ALLHAT Collaborative Research Group. The Antihypertensive and Lipid-Lowering Treatment to Prevent Heart Attack Trial. Major outcomes in high-risk hypertensive patients randomized to angiotensin-converting enzyme inhibitor or calcium channel blocker vs diuretic: the Antihypertensive and Lipid-Lowering Treatment to Prevent Heart Attack Trial (ALLHAT). JAMA 2002;288:2981-2997. [PubMed: 12479763]

27. Dahlöf B, Devereux RB, Kjeldsen SE, Julius S, Beevers G, de Faire U, Fyhrquist F, Ibsen H, Kristiansson K, Lederballe-Pedersen O, Lind-holm LH, Nieminen MS, Omvik P, Oparil S, Wedel H. LIFE Study Group. Cardiovascular morbidity and mortality in the Losartan Intervention For Endpoint reduction in hypertension study (LIFE): a randomised trial against atenolol. Lancet 2002;359:995-1003. [PubMed: 11937178]

28. Pitt B, Pfeffer MA, Assmann SF, Boineau R, Anand IS, Claggett B, Clausell N, Desai AS, Diaz R, Fleg JL, Gordeev I, Harty B, Heitner JF, Kenwood CT, Lewis EF, O’Meara E, Probstfield JL, Shaburishvili T, Shah SJ, Solomon SD, Sweitzer NK, Yang S, McKinlay SM. TOPCAT Investigators. Spironolactone for heart failure with preserved ejection fraction. N Engl J Med 2014;370:1383. [PubMed: 24716680]

29. Baumgartner H, Falk V, Bax JJ, De Bonis M, Hamm C, Holm PJ, Iung B, Lancellotti P, Lansac E, Muñoz DR, Rosenhek R, Sjögren J, Tornos Mas P, Vahanian A, Walther T, Wendler O, Windecker S, Zamorano JL. 2017 ESC/EACTS guidelines for the management of valvular heart disease: the task force for the management of valvular heart disease of the European Society of Cardiology (ESC) and the European Association for Cardio-Thoracic Surgery (EACTS). Eur Heart J 2017. 
30. Authors/Task Force membersElliott PM, Anastasakis A, Borger MA, Borggrefe M, Cecchi F, Charron P, Hagege AA, Lafont A, Limongelli G, Mahrholdt H, McKenna WJ, Mogensen J, Nihoyannopoulos P, Nis-tri S, Pieper PG, Pieske B, Rapezzi C, Rutten FH, Tillmanns C, Watkins H. 2014 ESC guidelines on diagnosis and management of hypertrophic cardiomyopathy: the task force for the diagnosis and management of hypertrophic cardiomyopathy of the European Society of Cardiology (ESC). Eur Heart J 2014;35:2733-2779. [PubMed: 25173338]

31. Ha JW, Oh JK, Ling LH, Nishimura RA, Seward JB, Tajik AJ. Annulus paradoxus: transmitral flow velocity to mitral annular velocity ratio is inversely proportional to pulmonary capillary wedge pressure in patients with constrictive pericarditis. Circulation 2001;104:976-978. [PubMed: 11524387]

32. Pereira NL, Grogan M, Dec GW. Spectrum of restrictive and infiltrative cardiomyopathies: part 1 of a 2-part series. J Am Coll Cardiol 2018;13(71):1130-1148. 10.1016/j.jacc.2018.01.016. Review.

33. Pereira NL, Grogan M, Dec GW. Spectrum of restrictive and infiltrative cardiomyopathies: part 2 of a 2-part series. J Am Coll Cardiol 2018;71:1149-1166. 10.1016/j.jacc.2018.01.017. Review. [PubMed: 29519356]

34. Loboz-Grudzień K, Kowalska A, Brzezińska B, Sokalski L, Jaroch J. Early predictors of adverse left ventricular remodelling after myocardial infarction treated by primary angioplasty. Cardiol J 2007;14:238-245. [PubMed: 18651467]

35. Zile MR, Brutsaert DL. New concepts in diastolic dysfunction and diastolic heart failure: part II: causal mechanisms and treatment. Circulation 2002;105:1503-1508. [PubMed: 11914262]

36. Reddy YN, Melenovsky V, Redfield MM, Nishimura RA, Borlaug BA. High-output heart failure: a 15-year experience. J Am Coll Cardiol 2016;68:473-482. [PubMed: 27470455]

37. Obokata M, Reddy YNV, Pislaru SV, Melenovsky V, Borlaug BA. Evidence supporting the existence of a distinct obese phenotype of heart failure with preserved ejection fraction. Circulation 2017;136:6-19. 60. [PubMed: 28381470]

38. Carbone S, Canada JM, Buckley LF, Trankle CR, Dixon DL, Buzzetti R, Arena R, Van Tassell BW, Abbate A. Obesity contributes to exercise intolerance in heart failure with preserved ejection fraction. J Am Coll Cardiol 2016;68:2487-2488. [PubMed: 27908355]

39. Kitzman DW, Brubaker P, Morgan T, Haykowsky M, Hundley G, Kraus WE, Eggebeen J, Nicklas BJ. Effect of caloric restriction or aerobic exercise training on peak oxygen consumption and quality of life in obese older patients with heart failure with preserved ejection fraction: a randomized clinical trial. JAMA 2016;315:36-46. [PubMed: 26746456]

40. Pandey A, Parashar A, Kumbhani DJ, Agarwal S, Garg J, Kitzman D, Levine BD, Drazner M, Berry JD. Exercise training in patients with heart failure and preserved ejection fraction: metaanalysis of randomized control trials. Circ Heart Fail 2015;8:33-40. [PubMed: 25399909]

41. Seferović PM, Paulus WJ. Clinical diabetic cardiomyopathy: a two-faced disease with restrictive and dilated phenotypes. Eur Heart J 2015;36:1718-1727. 10.1093/eurheartj/ehv134. [PubMed: 25888006]

42. Kalogeropoulos AP, Butler J. Heart rate in heart failure with preserved ejection fraction: target or marker? Eur J Heart Fail 2017 10.1002/ejhf.923. [Epub ahead of print].

43. Coats AJS, Forman DE, Haykowsky M, Kitzman DW, McNeil A, Campbell TS, Arena R. Physical function and exercise training in older patients with heart failure. Nat Rev Cardiol 2017. 

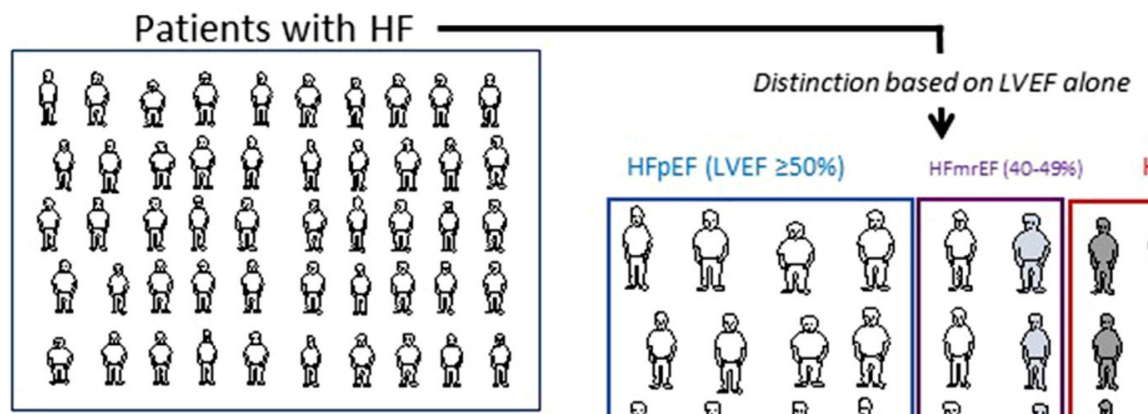

HFpEF (LVEF 250\%) HFmrEF (40-4956) HFrEF (LVEF<40\%)

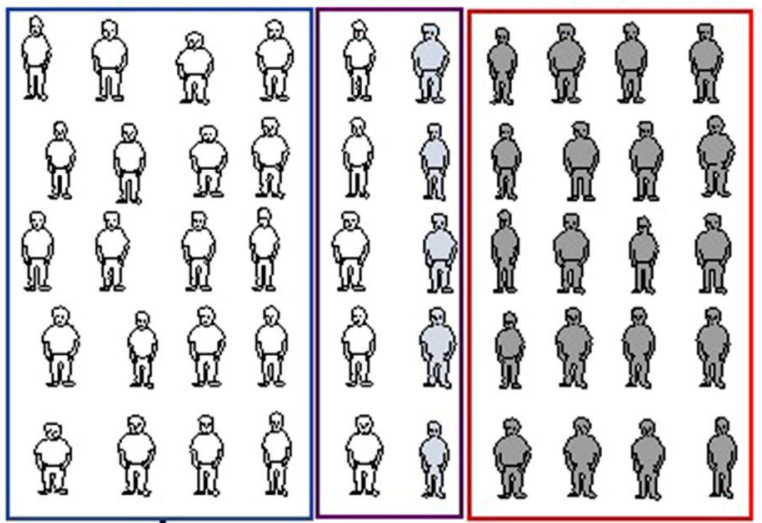

Distinction between

Primary and Secondary HFpEF

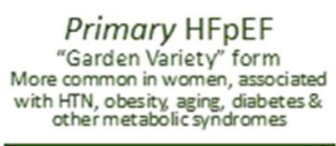

$\downarrow \quad$ Secondary HFpEF

A condition in which the diastolic he art failure is secondary to an impairment in the myocardium, valve or pericardium or an abnor mal cardiac rhythm

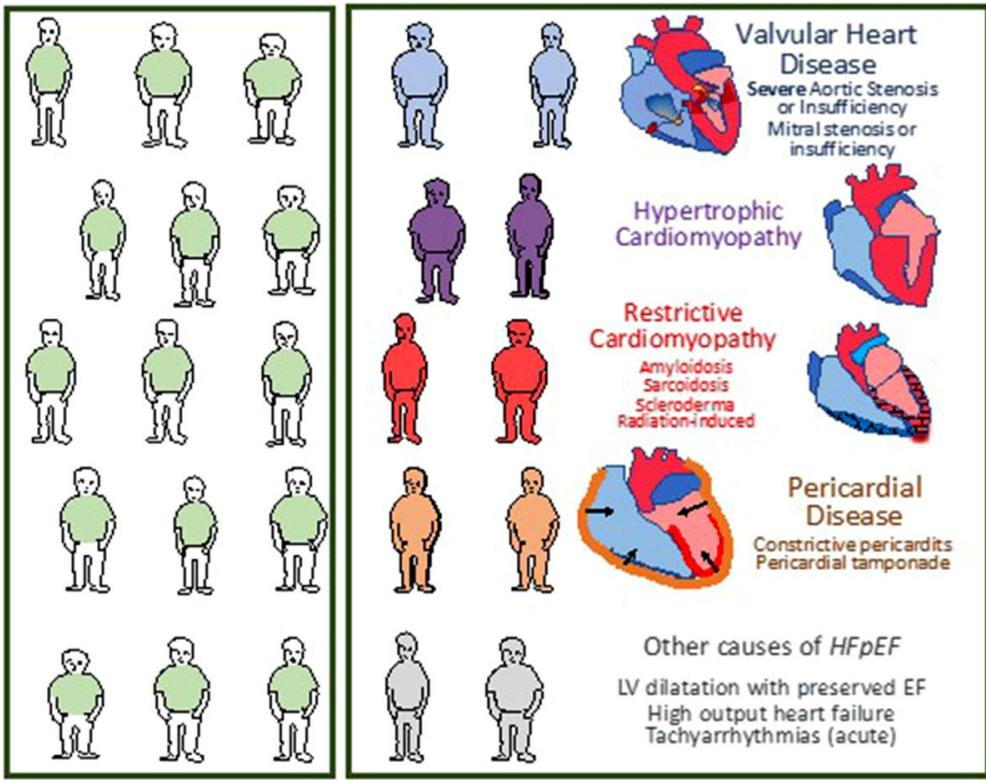

Figure 1.

Primary and secondary HFpEF. The exact prevalence of HFpEF is unknown. It is estimated at approximately $40 \%$ of patients with heart failure (HF) have reduced EF\% (LVEF $\$ 40 \%$ ), $20 \%$ have LVEF between $40 \%$ and $49 \%$, and the remaining have preserved left ventricular ejection fraction (LVEF $250 \%$ ) or HFpEF. Patients with HFpEF can be distinguished in 2 subgroups based on whether a specific cause can be identified or not. Secondary causes of HFpEF include valvular heart disease, hypertrophic cardiomyopathy, restrictive/infiltrative cardiomyopathies, constrictive pericarditis and other conditions. Primary HFpEF refers to a condition in which a primary impairment in myocardial relaxation or compliance exists. The 
prevalence of HFpEF in its primary and secondary forms is not represented in epidemiological scale. $\mathrm{HF}=$ heart failure; HFmEF: HE with mid-range ejection fraction; $\mathrm{HFpEF}=\mathrm{HF}$ with preserved ejection fraction; $\mathrm{HFrEF}=\mathrm{HF}$ with reduced ejection fraction . 
Clinical Diagnosis of Heart Failure

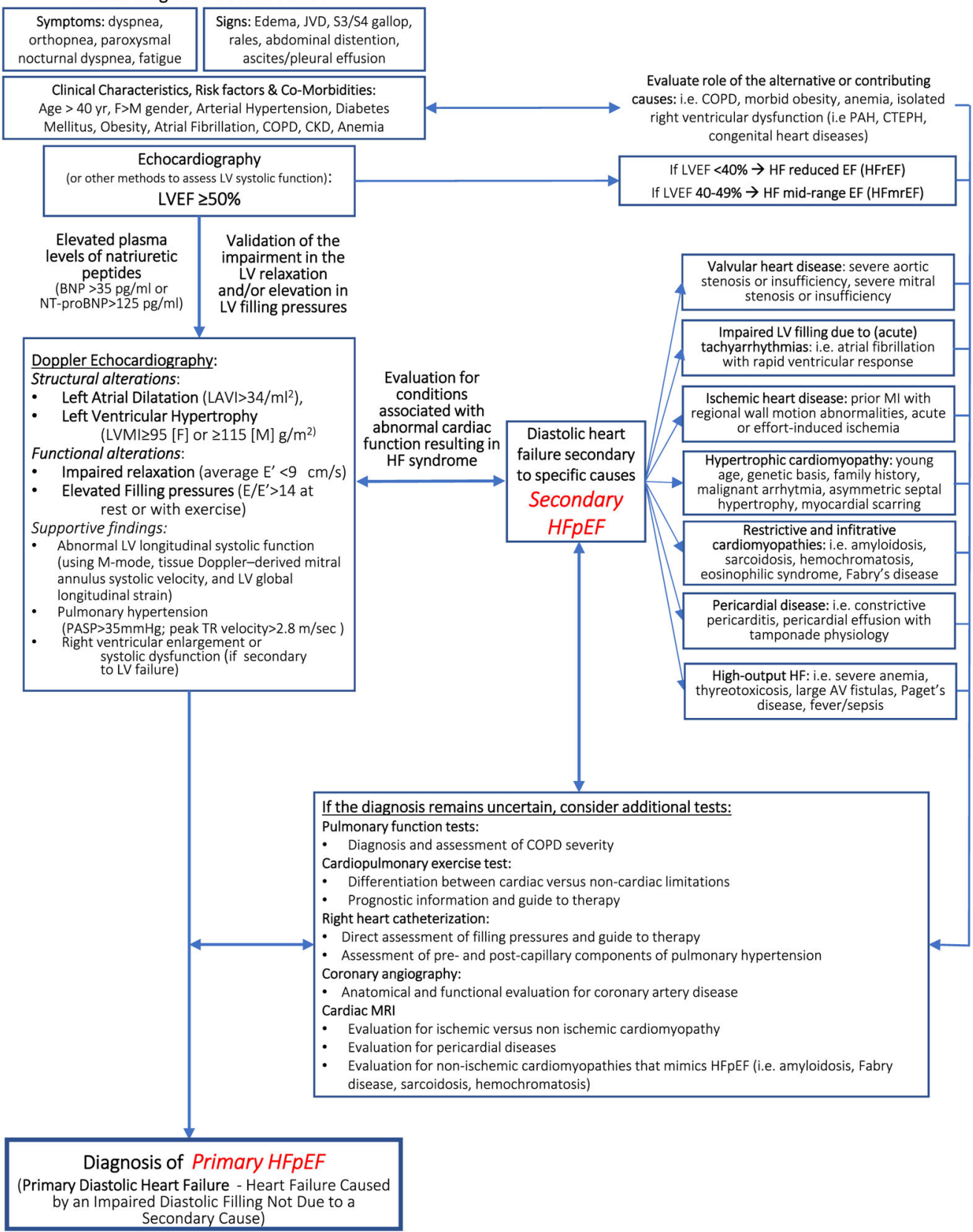

Figure 2.

Diagnostic algorithm for HFpEF. The diagnosis of HFpEF begins with the elicitation of symptoms and signs of heart failure, associated with the documentation of risk factors and co-morbid conditions. The diagnosis requires validation of the diagnosis of heart failure using data derived from either echocardio-Doppler study or invasive hemodynamics, or elevation of natriuretic peptides. Cardiac imaging is central to assessment as it allows to investigate the presence of the secondary causes HFpEF such as valvular heart disease, cardiomyopathies, pericardial disease, or other. In selected patients, additional tests may be necessary to further investigate the differential diagnosis of primary and secondary HFpEF, 
and to evaluate the role of concomitant non-cardiac disease that may present with similar symptoms and signs. $\mathrm{AF}=$ atrial fibrillation; $\mathrm{AV}=$ arteriovenous; $\mathrm{BNP}=$ brain natriuretic peptide; $\mathrm{CKD}=$ chronic kidney disease COPD = chronic obstructive pulmonary disease; $\mathrm{CTEPH}=$ chronic thromboembolic pulmonary hypertension; $\mathrm{DM}=$ diabetes mellitus; $\mathrm{EF}=$ ejection fraction; $\mathrm{F}=$ Female; $\mathrm{HF}=$ heart failure; $\mathrm{HFpEF}=$ heart failure with preserved ejection fraction; HTN = hypertension; JVD = jugular vein distension; LAVI = left atrial volume index; LV = left ventricle; $\mathrm{LVEF}=$ left ventricular ejection fraction; $\mathrm{LVMI}=$ left ventricular mass index; $\mathrm{M}=$ Male; $\mathrm{MI}=$ myocardial infarction; $\mathrm{NT}$-proBNP $=\mathrm{N}$-terminal pro brain natriuretic peptide; $\mathrm{PAH}=$ pulmonary arterial hypertension; $\mathrm{PASP}=$ pulmonary artery systolic pressure; $\mathrm{PH}=$ pulmonary hypertension. 


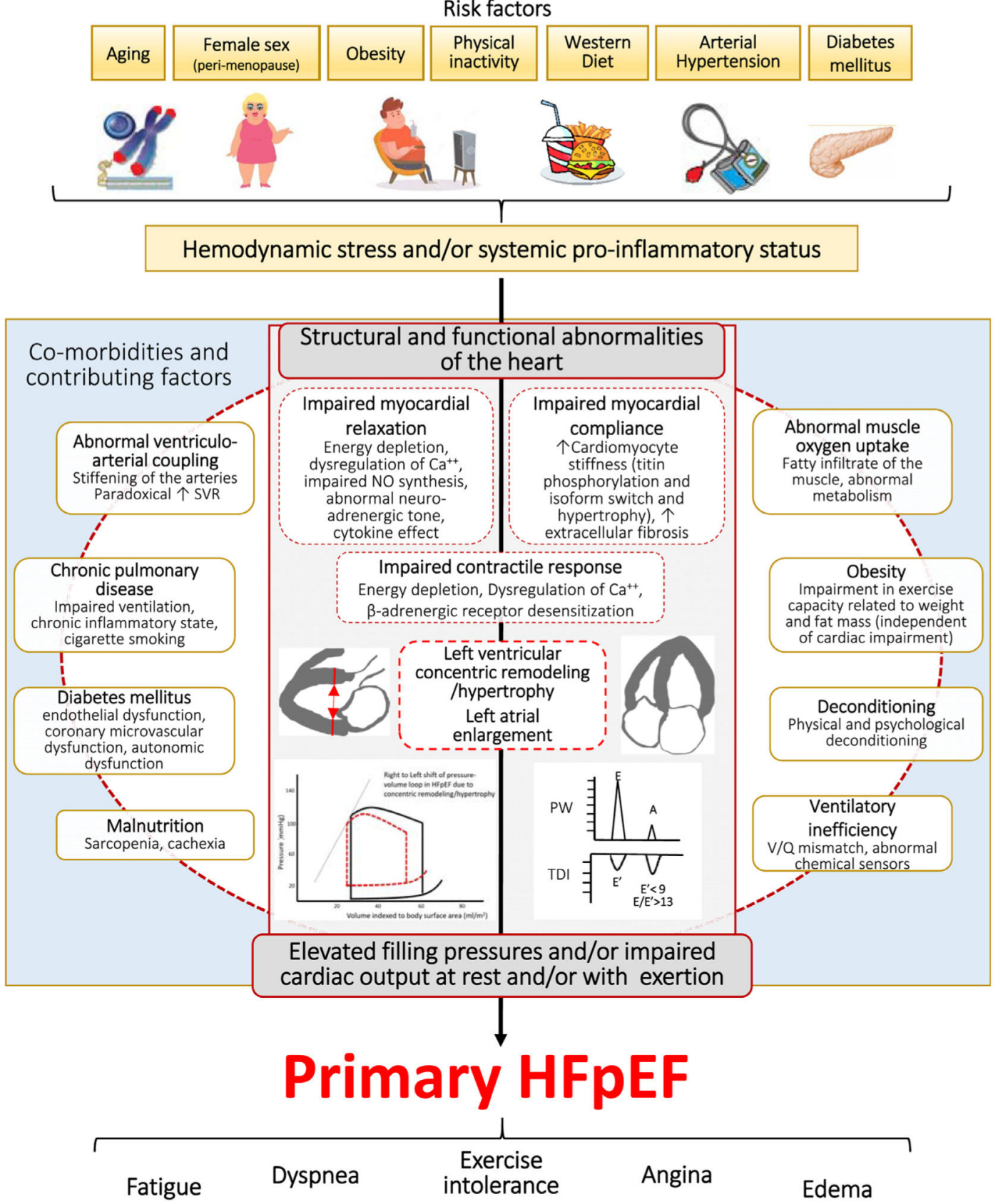

Figure 3.

Pathophysiology of primary HFpEF. Several risk factors (obesity, hypertension, aging, and physical inactivity) contribute to create a systemic proinflammatory status and/or haemodynamic stress that promote a primary myocardial diastolic dysfunction (impaired myocardial relaxation and compliance) leading to concentric remodeling/hypertrophy, and elevated LV filling pressures and/or impaired cardiac output. Moreover, co-morbid conditions and contributing factors (obesity, abnormal ventriculoarterial coupling, deconditioning, ventilatory inefficiency, pulmonary diseases, diabetes mellitus, and malnutrition) further contribute to exercise intolerance and clinical symptoms in patients 
with primary $\mathrm{HFpEF}$. HFpEF = heart failure with preserved ejection fraction; $\mathrm{LV}=$ left ventricle; $\mathrm{NO}=$ nitrogen oxygen $\mathrm{SVR}=$ systemic vascular resistance; $\mathrm{V} / \mathrm{Q}=$ ventilation/ perfusion ratio. 


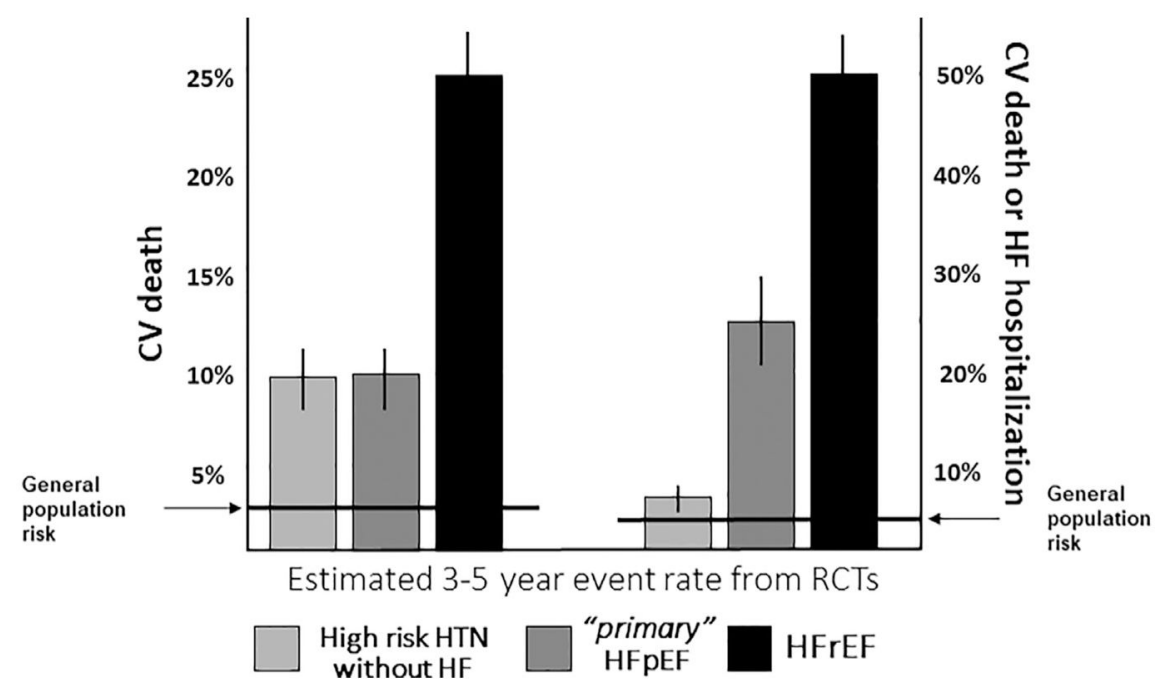

Figure 4.

Risk of CV death and CV death or HF hospitalization in high-risk patients with HTN without HF, patients with primary HFpEF and patients with HFrEF. The incidence of CV death at mid-term follow-up from randomized clinical trials appears to be lower in primary HFpEF compared with HFrEF, while not different than those with high-risk HTN without HF. The risk of HF hospitalization, in contrast, differs substantially between hypertensive patients without HF and those with HFpEF: patients with HFpEF have a much higher risk of $\mathrm{HF}$ hospitalization than patients with asymptomatic HTN. CV = cardiovascular; HFpEF = heart failure with preserved ejection fraction; $\mathrm{HFrEF}=$ heart failure with reduced ejection fraction; HTN = hypertension; RCT = randomized control trials. 


\section{Table 1}

Conditions that present with symptoms and signs of heart failure with preserved ejection fraction in which an identifiable cause is present (HFpEF).

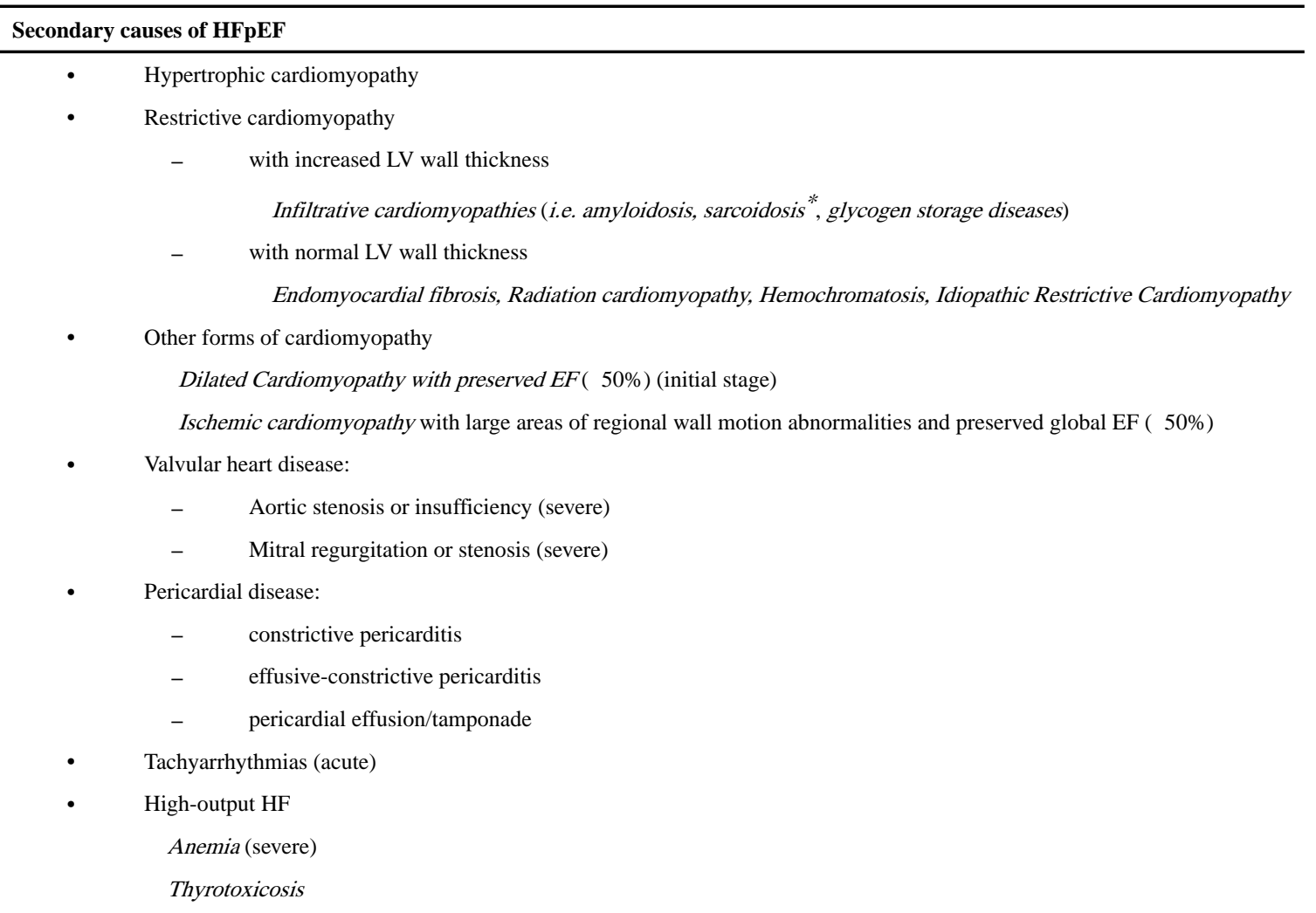

\footnotetext{
$\mathrm{ASD}=$ atrial septal defect EF = Ejection fraction; $\mathrm{LV}=$ left ventricle; $\mathrm{PA}=$ pulmonary atresia or pulmonary valvular stenosis; $\mathrm{VSD}=$ ventricula septal defect.

*ardiac sarcoidosis may present as restrictive cardiomyopathy with preserved EF (with increased or normal LV wall thickness) or as dilated cardiomyopathy with reduced EF.
} 